Published in final edited form as:

Hepatology. 2015 May ; 61(5): 1770-1771. doi:10.1002/hep.27430.

\title{
Response to: GLI2 as a Marker of Hedgehog-responsive Cells
}

\section{Cynthia Guy,}

Duke University, Pathology

\section{Ayako Suzuki,}

University of Arkansas

\author{
Manal Abdelmalek, \\ Duke University, Medicine Gastroenterology \\ James Burchette, and \\ Duke University Medical Center, Pathology
}

\author{
Anna Mae Diehl \\ Duke University, Divison of Gastroenterology and Hepatology
}

\section{Keywords}

Hedgehog signaling; Nonalcoholic steatohepatitis

We agree that nuclear localization of Gli2 protein can result from both canonical and noncanonical Hedgehog signaling. Our most recent study examined correlations between the severity of NASH and Hedgehog signaling activity in patients enrolled in the PIVENS trial. (1) Only a few liver sections were available for analysis from each subject. This limited the number of protein markers that could be examined and therefore, we restricted our analysis to those that have been proven to correlate well with Hedgehog pathway activity in prior studies. As Dr. Grzelak and her colleagues note, Hedgehog signaling results in the nuclear localization of Gli family proteins that regulate the mRNA expression of various Gli-target genes, including Gli1 and Ptc1. In our hands, commercially-available antibodies for Gli1 and Ptc1 perform inconsistently when used for immunohistochemical analysis of formalinfixed, paraffin-embedded human liver sections. Because our earlier studies demonstrated that various types of liver cells with nuclear-localized Gli2 protein express mRNAs for Hedgehog target genes, including Gli1 and Ptc1, we used Gli2 to mark potential Hedgehogresponsive cells in the NASH patients.

To further sub-classify the heterogeneous Gli2-positive population in the human livers, we co-stained for markers of myofibroblasts and liver progenitors because our prior work has shown that canonical Hedgehog signaling definitely occurs in these liver cell types. For example, almost a decade ago we demonstrated beta galactosidase activity in liver progenitors and stellate cells (the major source of myofibroblasts in NASH) from Ptc-LacZ reporter mice, proving that both cell types transcribe Ptc1, the receptor for Hedgehog ligands and a well-accepted target of canonical Hedgehog signaling. (2) Classically, Hedgehog ligands produced by epithelial cells interact with Ptc1 on the surface of neighboring stromal cells to initiate canonical Hedgehog signaling and activate Gli-regulated transcription in the 
Hedgehog-responsive stromal cells. We've reported that apoptotic and ER stress stimulate hepatocytes to produce and release biologically-active Hedgehog ligands. (3, 4) However, our earlier work also indicated that canonical Hedgehog signaling does not occur in mature hepatocytes themselves.(2) Thus, we agree that nuclear accumulation of Gli2 in hepatocytic cells must occur via one of the other mechanisms mentioned in Dr. Grzelak's letter. The fact that non-canonical Hedgehog signaling drives Gli2 localization in hepatocytes does not disprove that canonical Hedgehog signaling might be occurring in some other liver cell type(s), however.

If hepatocyte-derived Hedgehog ligands are activating canonical Hedgehog signaling in injured livers, this is likely to occur via the classical paracrine-mediated mechanism that targets stromal cells. Consistent with that concept, we shown that Hedgehog ligands stimulate hepatic stellate cells to become and remain myofibroblasts and demonstrated that this process coincides with increased stellate cell mRNA expression of Gli1, Ptc1, and other Hedgehog target genes. (5-8) Fluorescent-antibody cell sorting analysis confirmed that stellate cells also express these proteins, verifying that they are Hedgehog-responsive cells. Treating cultured stellate cells with anti-Hedgehog antibodies (to neutralize Hedgehog ligand activity), different pharmacologic inhibitors of Smoothened (an obligate component of the canonical Hedgehog signaling pathway), or using genetic approaches to delete Smoothened, inhibited expression of the Hedgehog target genes and prevented the myofibroblastic phenotype in cultured primary stellate cells. Identical responses were obtained in studies of different clonally-derived stellate cell lines and when targeted deletion of Smoothened was accomplished during various types of acute and chronic liver injury in mice (e.g., bile duct ligation, methionine choline deficient diet exposure, partial hepatectomy). $(8,9)$ In all of the in vivo experiments, the reduction in liver myofibroblasts that occurred when Hedgehog signaling was abrogated significantly improved liver fibrosis. Thus, a large body of published data indicates that injury-related activation of the canonical Hedgehog signaling pathway promotes liver fibrosis in rodents. Our latest study of NASH patients enrolled in the PIVENS trial suggests that similar mechanisms are operative in humans.(1) This is the main "take home message" of that work and it is important because the findings identify the Hedgehog pathway as a new target for biomarker development and therapeutics for NASH-related cirrhosis in humans.

\section{References}

1. Guy CD, Suzuki A, Abdelmalek MF, Burchette JL, Diehl AM. Treatment Response in the PIVENS Trial is Associated with Decreased Hedgehog Pathway Activity. Hepatology. 2014

2. Sicklick JK, Li YX, Melhem A, Schmelzer E, Zdanowicz M, Huang J, Caballero M, et al. Hedgehog signaling maintains resident hepatic progenitors throughout life. Am J Physiol Gastrointest Liver Physiol. 2006; 290:G859-870. [PubMed: 16322088]

3. Jung Y, Witek RP, Syn WK, Choi SS, Omenetti A, Premont R, Guy CD, et al. Signals from dying hepatocytes trigger growth of liver progenitors. Gut. 2010; 59:655-665. [PubMed: 20427400]

4. Rangwala F, Guy CD, Lu J, Suzuki A, Burchette JL, Abdelmalek MF, Chen W, et al. Increased production of sonic hedgehog by ballooned hepatocytes. J Pathol. 2011; 224:401-410. [PubMed: 21547909]

5. Sicklick JK, Li YX, Choi SS, Qi Y, Chen W, Bustamante M, Huang J, et al. Role for hedgehog signaling in hepatic stellate cell activation and viability. Lab Invest. 2005; 85:1368-1380. [PubMed: 16170335] 
6. Yang L, Wang Y, Mao H, Fleig S, Omenetti A, Brown KD, Sicklick JK, et al. Sonic hedgehog is an autocrine viability factor for myofibroblastic hepatic stellate cells. J Hepatol. 2008; 48:98-106.

[PubMed: 18022723]

7. Choi SS, Omenetti A, Witek RP, Moylan CA, Syn WK, Jung Y, Yang L, et al. Hedgehog pathway activation and epithelial-to-mesenchymal transitions during myofibroblastic transformation of rat hepatic cells in culture and cirrhosis. Am J Physiol Gastrointest Liver Physiol. 2009; 297:G10931106. [PubMed: 19815628]

8. Michelotti GA, Xie G, Swiderska M, Choi SS, Karaca G, Kruger L, Premont R, et al. Smoothened is a master regulator of adult liver repair. J Clin Invest. 2013; 123:2380-2394. [PubMed: 23563311]

9. Swiderska-Syn M, Syn WK, Xie G, Kruger L, Machado MV, Karaca G, Michelotti GA, et al. Myofibroblastic cells function as progenitors to regenerate murine livers after partial hepatectomy. Gut. 2014; 63:1333-1344. [PubMed: 24173292] 